\title{
THE COMPACT STEAM TURBINE -A NOVEL APPROACH TO SMALL SCALE COMBINED HEAT AND POWER GENERATION
}

\author{
Markus Kley ${ }^{\mathrm{a}}$, Rüdiger Kretschmer ${ }^{\mathrm{b}}$, Yorck Leschber ${ }^{\mathrm{b}}$, Ralf Rieger ${ }^{\mathrm{b}, *}$ \\ ${ }^{a}$ Hochschule Aalen, Beethovenstr. 1, 73430 Aalen, Germany \\ ${ }^{b}$ DHBC e.V., Schlossberg 12, 67366 Weingarten, Germany
}

\begin{abstract}
Current power plant technology needs to be simplified in order to be useful for small-scale systems. The small-scale turbine which represents an integrated small power plant originally invented at the Hochschule Darmstadt addresses this demand. Essentially, it is a very special application of a rotating heat pipe. It consists of rotating parts, thus reversing the traditional arrangement in a turbine. The device is divided by a plate into an evaporation and condensation chamber. This construction allows higher pressure differences so that a RHP can operate as a central part of a small power plant.

Keywords: Rotating Heat Pipe, Small-Scale Turbine, Micro CHP, solar thermal power.
\end{abstract}

\section{INTRODUCTION}

It was pointed out that small-scale CHP systems need to be simplified in order to be competitive with medium or large-scale systems (Dong et al., 2009). A novel approach is the Kompakte Dampf Turbine (KDT, meaning compact steam turbine), originally invented at the Hochschule Darmstadt, which addresses this demand (Heddrich et al., 2004). In this article, the compact steam turbine is examined in relation to a rotating heat pipe (RHP). The KDT can serve in a cogeneration plant (microCHP) to convert thermal energy into electricity, the remaining energy being used for heating. The KDT is about $30 \mathrm{~cm}$ in diameter and about $30 \mathrm{~cm}$ in height. With an additional external power generator it represents a complete power plant, not only a turbine. One big advantage of the KDT is its simplicity. For its construction only very few parts are necessary (Fig.1), namely an evaporator, a turbine which consists of a rotating cylinder with a rotating dividing plate and nozzles on it plus an optional blade wheel system. Through the rotational force the turbine also acts as a pump.

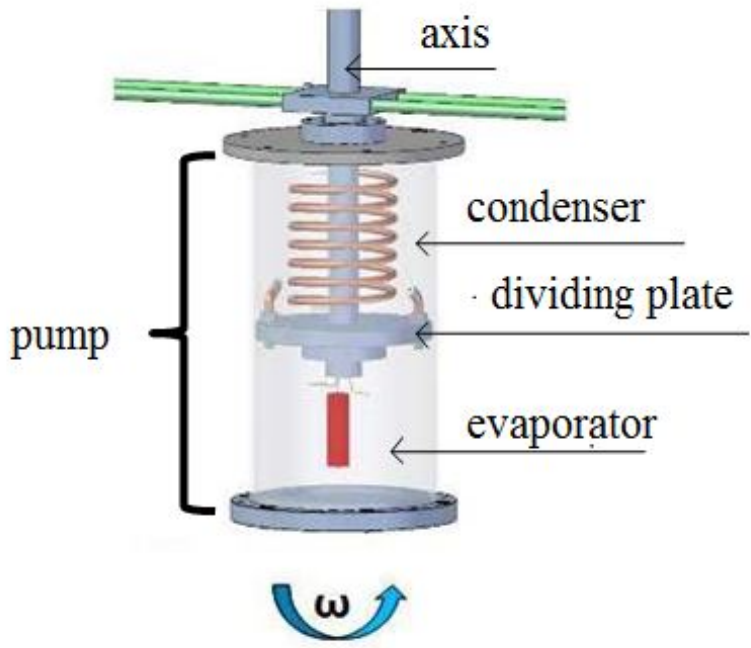

Fig. 1 Sketch of the KDT.

\section{THE NEWTONIAN BUCKET AS THE UNDERLYING MECHANICAL PRINCIPLE}

One of the crucial principles of the KDT is the "Newtonian Bucket". This term describes a cylinder partially filled with a liquid and set in rotation. Depending on the speed of rotation $\omega$, the free surface of the liquid is at first parabolic rapidly assuming the shape of an annular tube. The pressure on the inner wall is the sum of atmospheric pressure and the pressure due to the centrifugal force of the rotating liquid (Fig. 2).

The shape of the surface (eqs. (1) and (2)) is derived in many textbooks, for example Sigloch (2004).

$$
\begin{aligned}
& F_{g}=m g \quad F_{c}=m x \omega^{2} \\
& \frac{d y}{d x}=\frac{F_{c}}{F_{g}}=\frac{x}{g} \omega^{2} \quad \Rightarrow>y=\frac{\omega^{2}}{2 g} x^{2}
\end{aligned}
$$

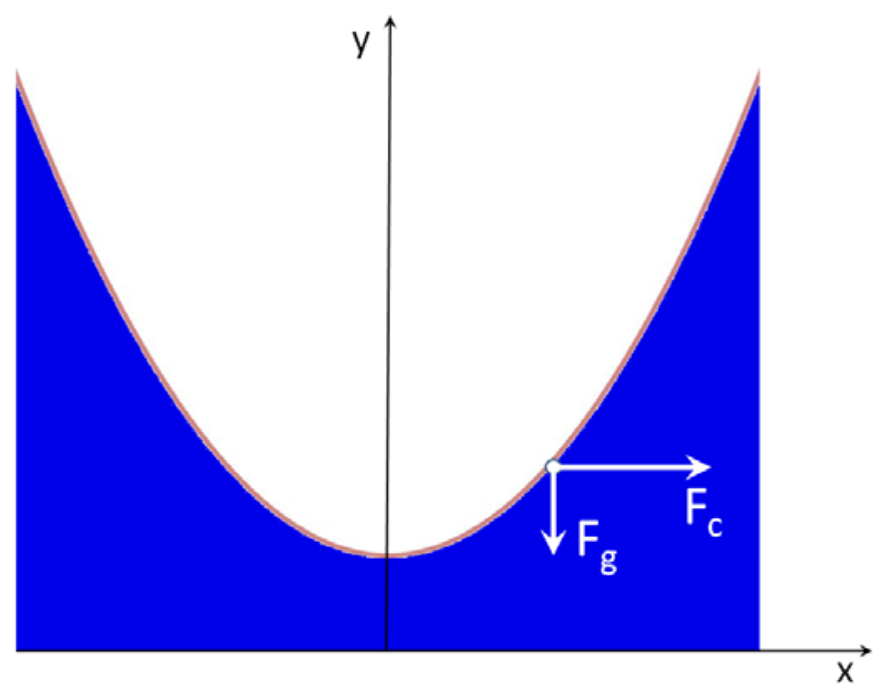

Fig. 2 The principle of "Newtonian bucket". 


\section{ROTATING HEAT PIPE}

As far as the heat transfer properties of the KDT are concerned the KDT is a variation of the rotating heat pipe (RHP). One attribute of the RHP are the large heat transfer coefficients. The RHP is a variation of the heat pipe: a heat transfer device that has been developed since the 1960s which is shown in Fig. 3. Gray (1969) formulated the initial proposal of a RHP and an overview is given by Reay and Kew (2006).

On the evaporator side the heat evaporates the liquid. The pressure on the high temperature side is higher than on the low temperature side resulting in a flow of the vapor to the condenser. The condensed liquid is returned to the evaporator side either by gravity (thermosyphon) or by the capillary (heat pipe) forces.

Since the vapor and liquid fluid reside in the same space, there are no large temperature and pressure differences in this RHP. Therefore the rotating heat pipe is not designed as thermal engine, where large pressure differences are necessary, despite some earlier considerations by Nguyen (T. Nguyen et al., 1999).

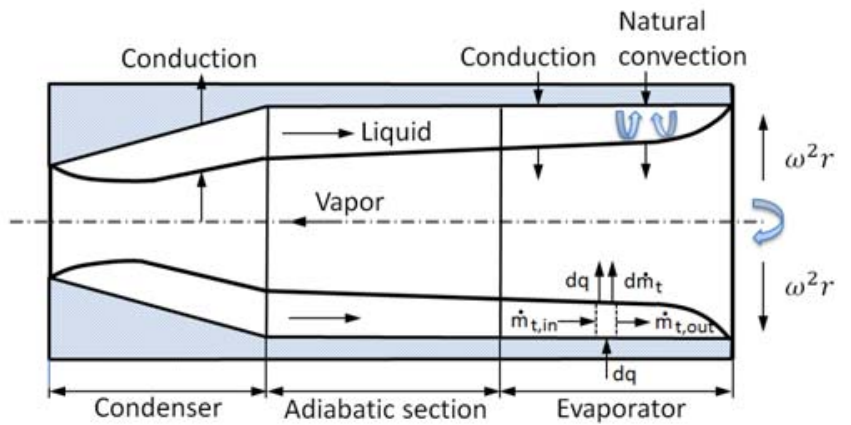

Fig. 3 Processes in the rotating heat pipe. Description is from Song (Song et al., 2003).

In a RHP the condensed fluid is returned as a result of the centrifugal forces. Sometimes the condenser end of an RHP has an inclination (taper angle). This form increases the centrifugal forces, i.e. improves the flow back to the evaporator.

\section{FROM RHP TO KDT}

The step from RHP to KDT is the introduction of a dividing plate. One can view the KDT as a rotating cylinder partially filled with liquid, divided by a plate. Between the edge of the plate and the inner wall of the cylinder there is a small gap. The "Newtonian Bucket" effect pushes the liquid up the cylinder wall, covering the gap. The liquid then seals the gap which has a width s, effectively insulating the upper and the lower chamber from each other (Fig. 4).

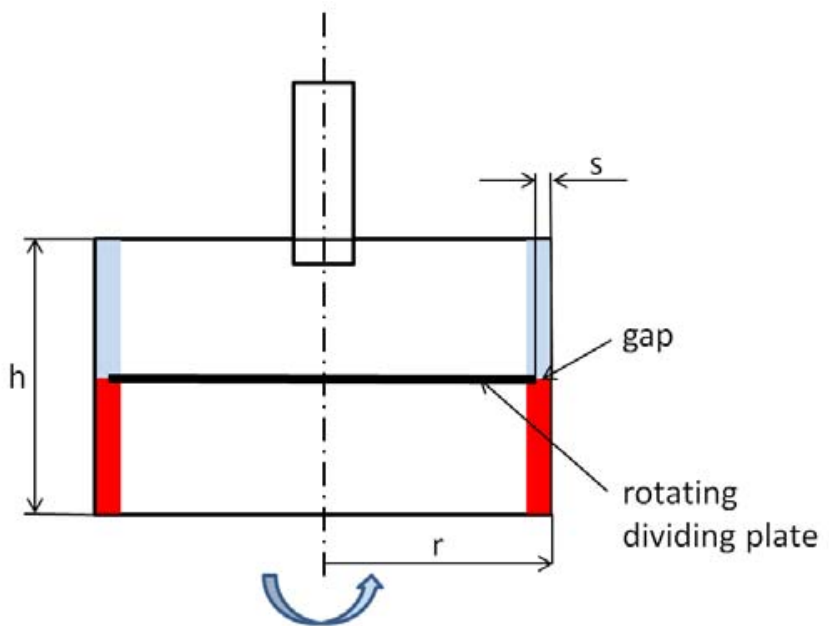

Fig. 4 Sketch of a fast rotating cylinder partially filled with liquid.
The total pressure on the inner cylinder wall is the sum of the gas pressure and the centrifugal pressure from the rotating liquid. If the gas pressure in the lower chamber is increased then there will be a new equilibrium. Part of the liquid is pushed through the gap in the upper chamber. The total pressure on the cylinder wall must be equal: In the lower chamber the gas pressure $\left(\mathrm{p}^{*}\right)$ is increased and the centrifugal pressure lowered. In the upper chamber the gas pressure (p) remains the same and the centrifugal pressure increases (since there is more liquid there), as shown in Fig. 5.

The rotating dividing plate has an important function: It effectively seals the chambers in collaboration with the liquid and therefore different gas pressures can be maintained in the chambers.

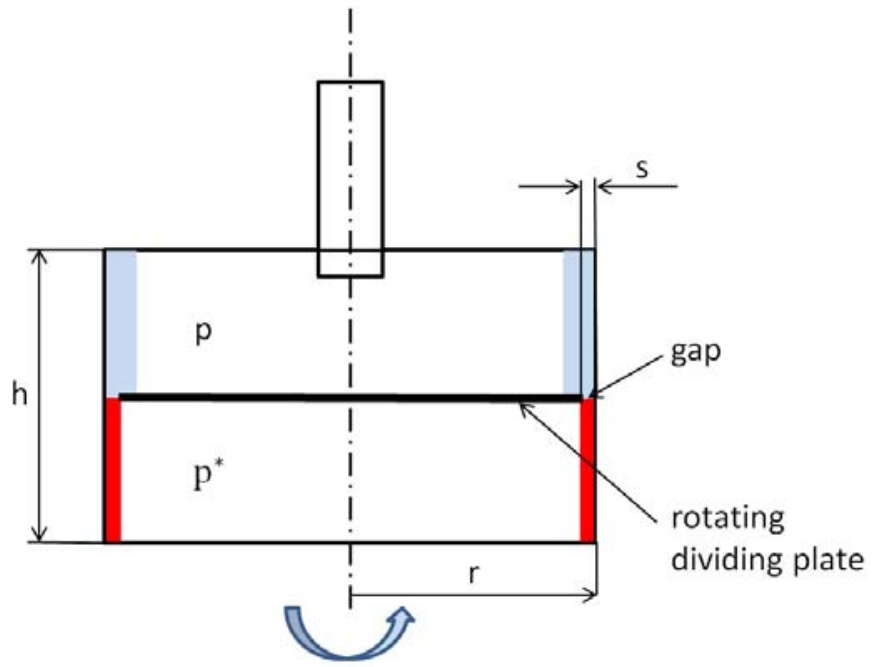

Fig. 5 Same as Fig.4 with different pressure in evaporator and condenser.
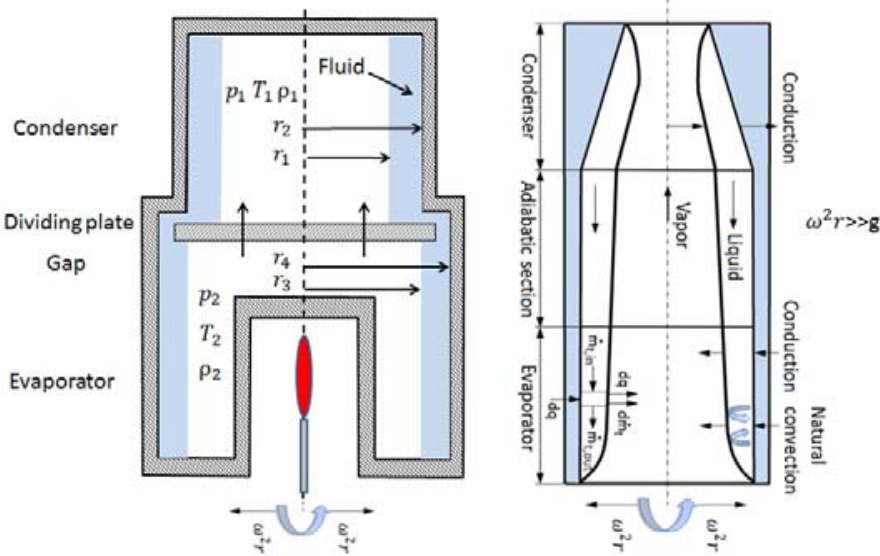

Fig. 6 Comparison between KDT and Rotating Heat Pipe.

In Fig. 6 a comparison between KDT and RHP is given. Here $p_{1}$ is the gas pressure in the upper chamber, $\mathrm{p}_{2}$ in the lower chamber. The radius $r_{1}$ is the distance of the fluid surface from the center in the upper chamber, $r_{3}$ in the lower chamber. $r_{2}$ and $r_{4}$ are the radii of the upper chamber and lower chamber. $T_{1}$ is temperature of the condensate, $T_{2}$ temperature of the vapor. The right side of Fig. 6 is from Song et al (2003).

If one now introduces holes in the partitioning plate allowing for vapor transfer from one chamber to another, one can see the similarity between the RHP and the KDT. The lower chamber serves as evaporator and the upper chamber as condenser.

In a traditional RHP as shown in Fig. 3 only relatively small pressure gradients between evaporator and condenser are possible. 
These are typically a few Kelvin and a few hundred Pascal (Song et. al., 2003). In contrast, the KDT can support temperature differences of several hundred Kelvin and pressure gradients of several MPa. The reason for this difference is that the dividing plate together with the rotating fluid effectively seals the two chambers from each other.

In the KDT the nozzles serve as the first stage of a propulsion system which can be enhanced by suitable turbine blades. There is of cause a crucial difference between the KDT and an RHP: The KDT will generate power not merely transport heat.

\section{CLAUSIUS RANKINE CYCLE FOR THE KDT}

In principle the KDT can use any working fluid. However, for developing the first prototypes we concentrate on dry Organic Rankine Cycle fluids (ORC), like pentane, toluene or butylbenzene which have a number of thermodynamic advantages at low temperatures. The visualization of the Organic Rankine Cycle is given in Fig. 7.

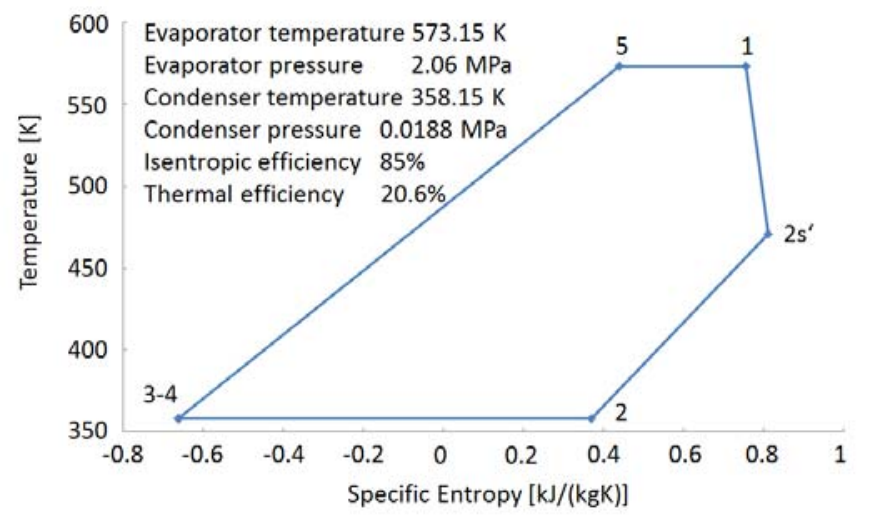

Fig. 7 T-S diagram for Organic Rankine Cycle (p-xylene) for typical temperatures of a CHP.

The Calculation is based on Fluidprop (Colonna et al., 2004). To simplify the description reference is given to the study by Liu where a simple ideal ORC is evaluated (Liu et al., 2004). The main five processes include the increase of pressure by pumping $(3 \rightarrow 4$ rotating KDT with angular velocity $\omega)$, heating by evaporator $(4 \rightarrow 5$, e.g. light input in Fig. 8), evaporating $(5 \rightarrow 1)$, providing work output via expander $\left(1 \rightarrow 2 s^{\prime}\right.$, nozzles in Fig. 8$)$, and cooling by condenser $\left(2 s^{\prime} \rightarrow 2\right.$, cooler in Fig 8) and condensing $(2 \rightarrow 3$, cooler in Fig 8).

The new aspect is that this cycle takes place in a modified RHP with high pressure difference, so that the weak performance described by Nguyen et al., (1999) should be overcome.

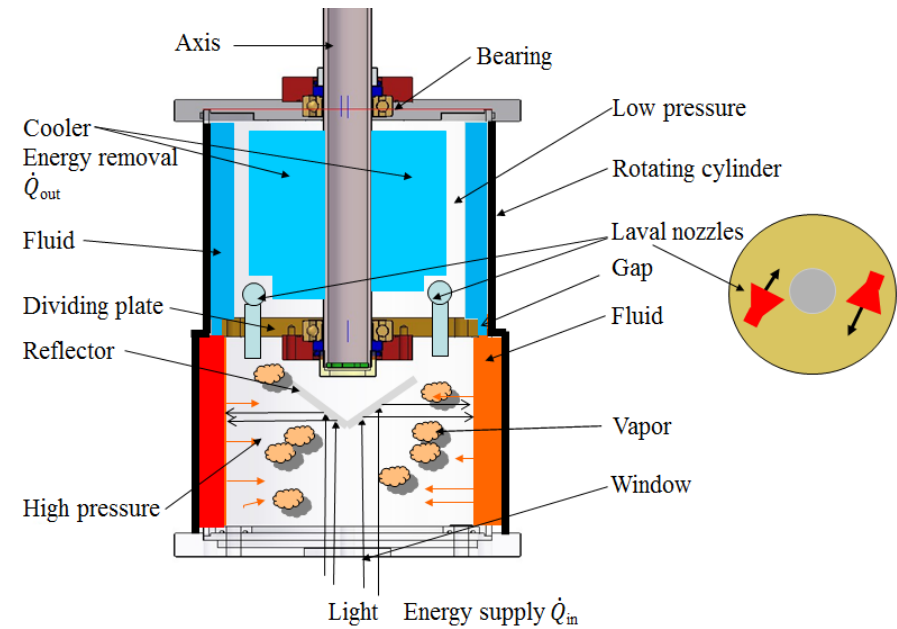

Fig. 8 The Organic Rankine Cycle in the KDT.
In Fig. 8 the complete KDT is shown. Heating, evaporation, expansion, condensation and pumping is embedded in a rotating, cylindrical device. Energy for heating the working fluid can be provided via conventional fuel like gas or via regenerative energy like solar heating.

A calculation has been carried out for p-xylene and is shown in Table 1 and plotted in Fig 7. The calculation is based on Fluidprop (P. Colonna and T.P. van der Stelt, 2004). More accurate results are from Lai et al., (2011) which result in a thermal efficiency of $20.4 \%$. For this rough estimation and discussion of the KDT principle a high accuracy is not needed.

In Table 1 the calculations for Fig. 7 are presented. The calculations are normalized to $20 \mathrm{~kW}$. The mass flows of the ORC fluids under consideration are in the neighborhood of $30-50 \mathrm{~g} / \mathrm{s}$ whereas water would only yield $<10 \mathrm{~g} / \mathrm{s}$ due to its large latent heat.

Table 1: Calculations for Fig. 7

\begin{tabular}{|l|l|l|l|l|}
\hline State & $\begin{array}{l}\text { Spec. Enthalpy } \\
{[\mathrm{kJ} / \mathrm{kg}]}\end{array}$ & $\begin{array}{l}\text { Spec. Entropy } \\
{[\mathrm{kJ} /(\mathrm{kgK})]}\end{array}$ & $T[\mathrm{~K}]$ & $\begin{array}{l}\text { Pressure } \\
{[\mathrm{MPa}]}\end{array}$ \\
\hline 1 & 406.1 & 0.7554 & 573.15 & 2.06 \\
\hline $2 \mathrm{~s}$, & 260.7 & 0.8107 & 470.83 & 0.019 \\
\hline 2 & 77.9 & 0.3694 & 358.15 & 0.019 \\
\hline 3 & -290.7 & -0.6598 & 358.15 & 0.019 \\
\hline 4 & -289.0 & -0.6623 & 358.15 & 2.06 \\
\hline 5 & 224.3 & 0.4382 & 573.15 & 2.06 \\
\hline
\end{tabular}

\section{EQUILIBRIUM AND PUMPING OF THE KDT}

As described earlier the radii of the fluid surfaces in the condenser and evaporator are a result of the balancing from gas and centrifugal pressures. The details are shown in Fig. 6.

For a steady state in this special RHP we write down a simple equation for the total pressures in the condenser and the evaporator.

$$
p_{a 1}+\int_{p(r 1)}^{p(r 4)} d p=p_{a 2}+\int_{p(r 3)}^{p(r 4)} d p
$$

Using $d p=\rho \omega^{2} r d r$ results in:

$$
p_{a 1}+\int_{r 1}^{r 4} \rho_{1} \omega^{2} r d r=p_{a 2}+\int_{r 3}^{r 4} \rho_{2} \omega^{2} r d r
$$

Integrating and rearranging

$$
p_{a 2}-p_{a 1}=\frac{1}{2} \rho_{1} \omega^{2}\left(r_{4}^{2}-r_{1}^{2}\right)-\frac{1}{2} \rho_{2} \omega^{2}\left(r_{4}^{2}-r_{3}^{2}\right)
$$

Normalizing to $\rho_{1}$ describes a specific enthalpy difference.

$$
\frac{p_{a 2}-p_{a 1}}{\rho_{1}}=\frac{1}{2} \omega^{2}\left(r_{4}^{2}-r_{1}^{2}\right)-\frac{\rho_{2}}{2 \rho_{1}} \omega^{2}\left(r_{4}^{2}-r_{3}^{2}\right)
$$

Alternatively the equations can be derived from Gerber (1975).

In a power plant the Clausius Rankine Cycle has a state change which is correlated to some relatively small pumping power. The state change from $3 \rightarrow 4$ must be regarded (see Table 1). The increase in enthalpy results in needed pumping energy. The specific enthalpy increase $\Delta \mathrm{h}_{\mathrm{ts}}$ for $\mathrm{p}$-xylene is about $1700 \mathrm{~J} / \mathrm{kg}$. This enthalpy increase is relatively small and therefore not visible in a typical T-S diagram like Fig. 7. Introducing $\Delta \mathrm{h}_{\mathrm{ts}}$ into (6) results in:

$$
\frac{p_{a 2}-p_{a 1}}{\rho_{1}}+\Delta h_{t s}=\frac{1}{2} \omega^{2}\left(r_{4}^{2}-r_{1}^{2}\right)-\frac{\rho_{2}}{2 \rho_{1}} \omega^{2}\left(r_{4}^{2}-r_{3}^{2}\right)
$$


At a calculated mass flow of $0.027 \mathrm{~kg} / \mathrm{s}$ for $\mathrm{p}$-xylene at $\dot{\mathrm{Q}}_{\text {in }}$ of 20 $\mathrm{kW}$ delivers a pumping power of $46 \mathrm{~W}$ (see Table 2). The rotating system provides this small amount of power because a typical efficiency for small ORC turbines is about $10 \%$ (Mikielewicz et al., 2009; Belozerov et al., 2012). This corresponds to about $2.5 \%$ of the desired shaft power of about $2 \mathrm{~kW}$. Solving equation (7) for $r_{3}$ results in:

$$
r_{3}^{2}=\frac{2\left(p_{a 2}-p_{a 1}\right)}{\omega^{2} \rho_{2}}+\frac{\rho_{1}}{\omega^{2} \rho_{2}} \Delta h_{t s}-\frac{\rho_{1}}{\rho_{2}}\left(r_{4}^{2}-r_{1}^{2}\right)+r_{4}^{2}
$$

Equation (8) shows that the vapor pressures $\left(\mathrm{p}_{\mathrm{a} 1}, \mathrm{p}_{\mathrm{a} 2}\right)$ lead to different radii $r_{3}$ (and similarly $r_{1}$ ) of the liquid ring tubes in the evaporator and the condenser. Differences in the vapor pressure are compensated by corresponding geodesic pressures so that overall there is a pressure balance.

Table 2: Calculated fluid properties according to equation (8).

\begin{tabular}{|l|c|c|c|c|r|}
\hline Fluid & $\begin{array}{c}\text { Angular } \\
\text { velocity } \\
\omega[\mathrm{rad} / \mathrm{s}]\end{array}$ & $\begin{array}{c}\Delta p \\
=p_{2}-p_{1} \\
{[\mathrm{MPa}]}\end{array}$ & $\begin{array}{c}\rho \\
{\left[\mathrm{kg} / \mathrm{m}^{3}\right]} \\
\text { state 3 }\end{array}$ & $\begin{array}{c}\rho \\
{\left[\mathrm{kg} / \mathrm{m}^{3}\right]} \\
\text { state 4 }\end{array}$ & $\begin{array}{c}\text { Enthalpy } \\
\text { difference } \\
\Delta h_{3-4}[\mathrm{~J} / \mathrm{kg}]\end{array}$ \\
\hline $\mathrm{p}$-xylene & 1000 & 2.04 & 786.79 & 475.25 & 1700 \\
\hline water & 1000 & 0.74 & 968.69 & 969.02 & 330 \\
\hline
\end{tabular}

\begin{tabular}{|l|r|r|r|r|r|}
\hline Fluid & $r_{3}[\mathrm{~m}]$ & $\begin{array}{c}\text { Pumping } \\
\text { power } \\
\text { [Watt] }\end{array}$ & $\begin{array}{c}\text { Power } \\
\text { for } \\
\text { accele- } \\
\text { ration } \\
\text { [Watt] }\end{array}$ & $\begin{array}{c}\text { Mass- } \\
\text { flow } \\
{[\mathrm{kg} / \mathrm{s}]}\end{array}$ & $\begin{array}{c}\text { Thermal } \\
\text { efficiency } \\
{[\%]}\end{array}$ \\
\hline p-xylene & 0.1432 & 46 & 277 & 0.0270 & 20.6 \\
\hline water & 0.1500 & 2 & 71 & 0.0063 & 20.6 \\
\hline
\end{tabular}

Table 2 shows a listing of pressures, radii and densities for p-xylene and water. The heating takes place in the evaporator. Calculations were done with Fluidprop (Colonna et al. 2004). Here the pressures, radii and densities are listed. Exemplary insertion of values into (8) delivers results for p-xylene and water: the radii of KDT were set at $\omega=1000$ $\mathrm{rad} / \mathrm{s}$ to $\mathrm{r}_{1}=0.120 \mathrm{~m}$ and $\mathrm{r}_{2}=0.142 \mathrm{~m}, \mathrm{r}_{4}=0.150 \mathrm{~m}$. The assumption was to have equal thermal efficiencies.

In the static cooler it is necessary that the condensed working fluid is accelerated by the dividing plate. The required power for accelerating the fluid is indicated in Table 2 in the column "Power for acceleration". The required power is much less for water than it is for p-xylene as working fluid.

In a KDT the power for pumping provided by the rotating system is very low compared to the expected electrical power output. Under operational conditions a dynamical equilibrium is reached. If radii and rotational speed are suitably chosen then for given densities a solution at small pumping power is found where KDT simultaneously pumps and seals.

\section{CONCLUSIONS}

Previously the KDT was presented only as low-budget power source for micro-CHP. In this article it was shown that the working principle of the KDT is closely related to that of a RHP. This similarity allows us to exploit the research previously done on RHP. An example for typically required pumping power in the rotating system is given. The invention of Heddrich et al. (2003) is an approach to make the KDT as a special RHP feasible for use as a highly integrated and simple in construction Micro CHP for use with solar, regenerative (e.g. biofuels) and conventional energy sources.

The KDT is essentially a fully integrated power plant with numerous advantages: Very few parts, no valves, a liquid seal between condenser and evaporator, allowing for a competitive realization and low maintenance costs. The final goal is to develop a small scale robust integrated power plant which could be produced in a serial production for a small amount of money.

\section{NOMENCLATURE}

$c_{p} \quad$ specific heat $(\mathrm{J} / \mathrm{kg} \cdot \mathrm{K})$

$h \quad$ latent heat of phase change $(\mathrm{J} / \mathrm{kg})$

$k \quad$ thermal conductivity $(\mathrm{W} / \mathrm{m} \cdot \mathrm{K})$

$p \quad$ pressure $\left(\mathrm{kg} / \mathrm{m} \cdot \mathrm{s}^{2}\right)$

$t \quad$ time (s)

$T \quad$ temperature (K)

$m \quad$ mass $(\mathrm{kg})$

$r \quad$ radius $(\mathrm{m})$

$x \quad \mathrm{x}$-coordinate $(\mathrm{m})$

$y \quad \mathrm{y}$-coordinate $(\mathrm{m})$

$\dot{Q}_{\text {in }} \quad$ energy supply

$\dot{Q}_{\text {out }} \quad$ energy removal

\section{Greek Symbols}

$\rho \quad$ density $\left(\mathrm{kg} / \mathrm{m}^{3}\right)$

$\omega \quad$ angular velocity $(\mathrm{rad} / \mathrm{s})$

Subscripts

$g \quad$ gravitational component

c centrifugal component

\section{REFERENCES}

Belozerov, A., Kley, M., Leschber, Y., Rieger, R., Shatalov, I., Wiedmann, M., 2012, "A New Integrated Power Plant with a SmallScale Turbine for the Organic Rankine Cycle," RUDN Bulletin 2012 No. 3, pp. 104-113.

Colonna P., van der Stelt, T.P, 2004, FluidProp: a Program for the Estimation of Thermo Physical Properties of Fluids, Energy Technology Section, Delft University of Technology. http://www.FluidProp.com

Dong L., Liu, H., and Riffat, S., 2009, "Development of Small-Scale and Micro-Scale Biomass-Fuelled CHP Systems," Appl. Thermal Eng., 29, $2119-2126$.

http://dx.doi.org/10.1016/j.applthermaleng.2008.12.004

Gerber, N, 1975, Rigidly Rotating Liquids in Closed Partially-Filled Cylindrical Cavities, Ballistic Research Laboratories Report No. 2462.

Gray, V.H., 1969, “The Rotating Heat Pipe - A Wickless, Hollow Shaft for Transferring Heat Fluxes," Proc. ASME/AIChE Heat Transfer Conf., Minneapolis, pp. 1-5, ASME Paper No. 69-HT-19.

Heddrich, W., Lautner, H., Hein, L., Losch, M., 2004, Thermal Power Machine for Converting Thermal Energy into Mechanical Energy and Use Thereof, Patent DE10315746, 2004.

Lai, N. A., Wendland, M., Fischer, J., 2011, "Working Fluids for HighTemperature Organic Rankine Cycles," Energy, 36, 199-211. http://dx.doi.org/10.1016/j.energy.2010.10.051

Liu, B.-T., Chien, K.H., Wang, C.C., 2004, "Effect of Working Fluids on Organic Rankine Cycle for Waste Heat Recovery," Energy, 29, 1207-1217.

http://dx.doi.org/10.1016/j.energy.2004.01.004

Mikielewicz, J., Piwowarski, M., Kosowski, K., 2009, "Design Analysis of Turbines for Co-Generating Micro-Power Plant Working according to Dance with Organic Rankine's Cycle," Polish Maritime Research, 16, Special issue, 34-38.

http://dx.doi.org/10.2478/v10012-008-0042-4 
Nguyen, T., Mochizuki, M., Mashiko, M., Sauciuc, I., Akbarzadeh, A., Johnson, P., Kusaba, S., Suzuki, H., 2009, "Heat Pipe Turbine for Production of Electrical Power from Renewable Sources," Proceedings of 5th ASME/JSME Joint Thermal Engineering Conference, March 1519, 1999, San Diego, California

Reay, D. A., Kew, P. A., Heat Pipes, Amsterdam, Heidelberg, 2006, 5. ed.; XVII
Sigloch, H., 2004, Technische Fluidmechanik, Springer, 5.Auflage.

Song, F., Ewing, D., Ching, C. Y., 2003, "Fluid Flow and Heat Transfer Model for High-Speed Rotating Heat Pipes," International Journal of Heat and Mass Transfer, 46, 4393-4401.

http://dx.doi.org/10.1016/S0017-9310(03)00292-8 